\title{
Postnatal hydrocortisone treatment for chronic lung disease in the preterm newborn and long-term neurodevelopmental follow-up
}

\author{
K J Rademaker, ${ }^{1}$ L S de Vries, ${ }^{1}$ C S P M Uiterwaal, ${ }^{2} \mathrm{~F}$ Groenendaal, ${ }^{1}$ D E Grobbee, ${ }^{2}$ \\ $\mathrm{F}$ van $\mathrm{Bel}^{1}$
}

${ }^{1}$ Department of Neonatology, University Medical Center Utrecht/Wilhelmina Children's Hospital, Utrecht, The

Netherlands; ${ }^{2}$ Julius Center for Health Sciences and Primary Care, University Medical Center Utrecht, Utrecht, The Netherlands

Correspondence to: Dr K J Rademaker, Department of Neonatology, KE 04.123.1,

University Medical Center Utrecht/Wilhelmina Children's Hospital, PO Box 85090, 3508 AB Utrecht, The Netherlands; k.rademaker@umcutrecht.nl

Accepted 24 July 2007 Published Online First 11 September 2007

\section{ABSTRACT}

The benefits versus the risks of postnatal administration of steroids in preterm-born infants are still debatable. This review examines the literature on postnatal hydrocortisone treatment for chronic lung disease (CLD) in pretermborn infants with a particular focus on the effects of such treatment on long-term neurodevelopmental outcomes. Quantitative published evidence does not point to a clear advantage of treatment with hydrocortisone over dexamethasone with regard to the impact on long-term neurological outcomes. However, in the absence of a randomised comparison, a consensus may soon have to be reached on the basis of the best available evidence whether hydrocortisone should replace dexamethasone in the treatment of CLD.

In contrast with the consensus about the benefits of maternal steroid treatment to accelerate fetal lung maturation, ${ }^{1}$ there is an ongoing debate about the pros and cons of postnatal corticosteroid administration to preterm-born infants. ${ }^{2-6}$ After initial reports in the 1980s suggested short-term benefits of steroids in ventilator-dependent children, dexamethasone became the widely and almost exclusively used drug for preventing or treating chronic lung disease (CLD). ${ }^{7}$ One retrospective study examining the outcome of neonates with a birth weight between $500 \mathrm{~g}$ and $749 \mathrm{~g}$, showed that $43 \%$ of infants born between 1990 and 1992 received dexamethasone compared with as many as 84\% of those born between 1993 and 1995..$^{8}$ The almost routine use of dexamethasone continued until 1998, when Yeh et al published the results of a large multicentre follow-up study that showed a marked increase in neurodevelopmental dysfunction in neonates treated with dexamethasone compared with controls. ${ }^{9}$ More alarming publications on the long-term negative effects of dexamethasone appeared ${ }^{10}{ }^{11}$ and as a result there was a gradual decrease in postnatal steroid prescription. $^{12}$ The American Academy of Pediatrics stated in 2002 that, outside clinical trials, postnatal steroid use should be reserved only for "exceptional clinical circumstances". ${ }^{13}$ However, a recent prospective evaluation of postnatal steroid administration in California, from April 2002 to March 2003, showed that $19.3 \%$ of children $\leqslant 1500$ g were still receiving steroids. ${ }^{14}$

Over the past years, there have been more reports of long-term negative neurodevelopmental sequelae following neonatal dexamethasone treatment, ${ }^{9-11} 1516$ and recently an MRI study at term equivalent age showed reduced brain volumes in infants treated with a moderately low dose of dexamethasone after 28 days of life. ${ }^{17}$

Hydrocortisone may be an alternative to dexamethasone if it has fewer negative long-term side effects, but there have been scarcely any studies on long-term outcome after postnatal use of hydrocortisone in preterm infants. Hydrocortisone is also being increasingly used for treatment or prevention of vasopressor-resistant hypotension in neonatal medicine. ${ }^{18-20}$ However, besides one abstract presented at the Society for Pediatric Research meeting in 2007, ${ }^{21}$ there are no long-term developmental outcome data for infants treated with hydrocortisone for this indication.

The aim of this review was to summarise the literature on postnatal hydrocortisone treatment for CLD in preterm-born infants with a special focus on long-term neurodevelopmental outcome following this treatment. We will not discuss hydrocortisone treatment for refractory hypotension, as it is beyond the scope of this review.

\section{HYDROCORTISONE AND CHRONIC LUNG DISEASE}

Six reports have been published on postnatal hydrocortisone administration for prevention $(n=5)$ or treatment $(n=1)$ of CLD (summarised in table 1). The first randomised placebo controlled trial of hydrocortisone treatment was published as early as in $1972 .^{22}$ This trial evaluated the ability of postnatally administered hydrocortisone to alter the course of outcome in infants with hyaline membrane disease. In all, 44 infants (mean gestational age 32.5 weeks) were treated with hydrocortisone or a lactose placebo within $24 \mathrm{~h}$ after birth. There was no remarkable effect on $\mathrm{PaO}_{2}$, $\mathrm{PaCO}_{2}$, need for assisted mechanical ventilation or survival.

No other studies on hydrocortisone and respiratory disease were published hereafter until 1999, when Watterberg et al enrolled 40 preterm infants into a randomised pilot study to test whether early treatment with low-dose hydrocortisone for 12 days, started within $48 \mathrm{~h}$ after birth would increase the likelihood of survival without CLD. ${ }^{23}$ The rationale for this study was that many extremely low birthweight infants show biochemical evidence of adrenal insufficiency in the first week of life, correlating with subsequent development of CLD. ${ }^{24}$ Adrenal insufficiency is also associated with amplified inflammatory responses, because cortisol is essential for resolution of inflammation. Infants who develop CLD have 
Table 1a Hydrocortisone and chronic lung disease

\begin{tabular}{|c|c|c|c|c|c|c|c|}
\hline Study no. & Study & Type of study & No of patients & Treatment allocation & Mean GA & Mean BW & HC indication \\
\hline \multirow[t]{2}{*}{1} & Baden et $a_{1}{ }_{,}^{22} 1972$ & Randomised, placebo controlled & 44 & $22 \mathrm{HC}$ & 32.5 & 1730 & Prophylaxis \\
\hline & & & & 22 placebo & 31.8 & 1767 & \\
\hline \multirow[t]{2}{*}{2} & Watterberg et al, ${ }_{1}^{23} 1999$ & $\begin{array}{l}\text { Randomised, double-masked, } \\
\text { placebo controlled pilot }\end{array}$ & 40 & $20 \mathrm{HC}$ & 25.2 & 732 & Prophylaxis \\
\hline & & & & 20 placebo & 25.4 & 770 & \\
\hline \multirow[t]{2}{*}{3} & Heide-Jalving et al ${ }_{1}{ }^{26} 2003$ & Retrospective non-randomised & 50 & $25 \mathrm{HC}$ & 28.3 & 1040 & Treatment of CLD \\
\hline & & & & 25 control & 28.4 & 1090 & \\
\hline & & & & 180 placebo & 25.3 & 734 & \\
\hline \multirow[t]{2}{*}{5} & Peltoniemi et al ${ }_{1}^{28} 2005$ & Randomised, placebo controlled & Enrolment stopped at $51^{*}$ & $25 \mathrm{HC}$ & 26.7 & 888 & Prophylaxis \\
\hline & & & & 26 placebo & 26.5 & 903 & \\
\hline \multirow[t]{2}{*}{6} & Bonsante et $a_{1}{ }_{1}^{29} 2007$ & Randomised, placebo controlled & Enrolment stopped at $50 \dagger$ & $25 \mathrm{HC}$ & 26.2 & 840 & Prophylaxis \\
\hline & & & 25 placebo & 26.5 & 900 & & \\
\hline
\end{tabular}

Table 1b Hydrocortisone and chronic lung disease

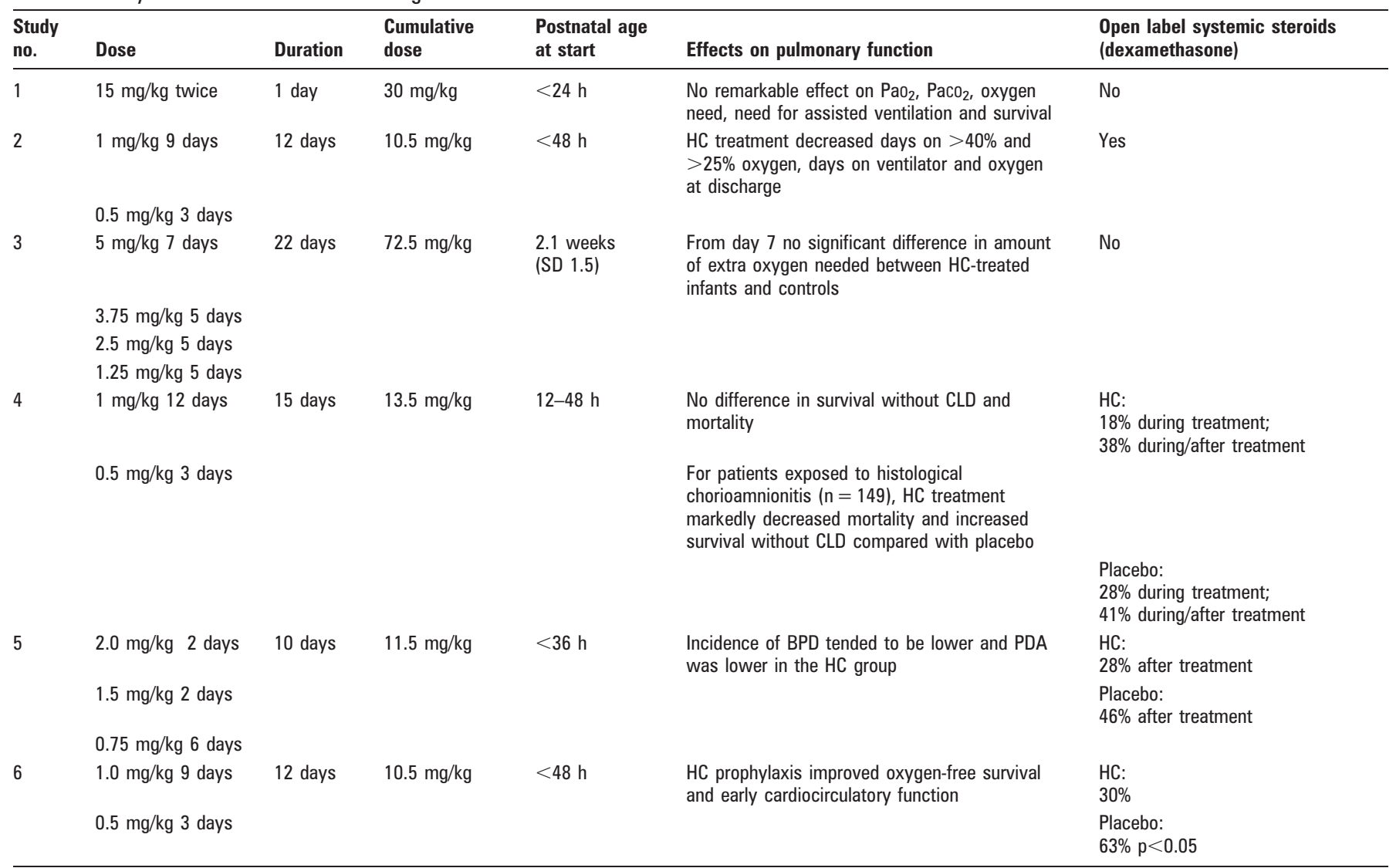

been shown to have raised levels of indicators of both prenatal and postnatal inflammation. ${ }^{25}$ Among the infants treated with hydrocortisone, $60 \%$ survived without supplemental oxygen at 36 weeks' postmenstrual age in contrast with $35 \%$ in the placebo group. Treatment with hydrocortisone decreased the number of days on $>40 \%$ oxygen, days on $>25 \%$ oxygen, days on ventilator and oxygen at discharge. Although fewer children in the hydrocortisone group were subsequently treated with dexamethasone, there was no difference in median days of dexamethasone administration between the two groups. Adverse short-term neonatal complications were similar for the two groups.

A retrospective matched-cohort study compared 25 preterm infants who were treated for CLD with a much higher dose of hydrocortisone, with 25 controls from the same centre. It showed equal reduction in the need for extra oxygen and successful weaning from the ventilator, similar to the results of another study conducted at a different centre that compared 23 infants receiving dexamethasone with 23 controls. ${ }^{26}$ The hydrocortisone and non-steroid groups did not differ with regard to mean arterial blood pressure, blood glucose, weight gain or spontaneous gastrointestinal perforation.

The multicentre trial following the 1999 pilot study ${ }^{23}$ enrolled mechanically ventilated infants with a birth weight of 500$999 \mathrm{~g}$ between $12 \mathrm{~h}$ and $48 \mathrm{~h}$ of life..$^{27}$ Enrolment stopped at 360 infants because of an increase in spontaneous gastrointestinal perforation in the hydrocortisone-treated group. For the total population, prophylactic treatment of early adrenal insufficiency 
did not improve survival without CLD or mortality at 36 weeks. However, for the patients exposed to histologically confirmed chorioamnionitis, hydrocortisone did markedly decrease mortality and increase survival without CLD. The study suggested an interactive effect between hydrocortisone and indometacin administered simultaneously, as a much higher percentage of these children had spontaneous gastrointestinal perforation.

Peltoniemi et al investigated the effect of early hydrocortisone treatment on survival without CLD in infants $\leqslant 30$ weeks and noticed a tendency towards lower CLD in the hydrocortisonetreated group. ${ }^{28}$ This study was also discontinued early after inclusion of 51 infants due to a higher incidence of gastrointestinal perforation in the hydrocortisone group. Three of the four children in the hydrocortisone group who had this complication had been treated simultaneously with indometacin/ibuprofen. The infants treated with hydrocortisone who had serum cortisol concentrations above the median had a high risk of gastrointestinal perforation. Infants treated with hydrocortisone but with cortisol values below the median showed increased survival without bronchopulmonary dysplasia.

Finally, Bonsante et al conducted a double-blind, randomised, placebo controlled trial in 50 mechanically ventilated infants (birth weight 500-1249 g) (see table 1 for details). ${ }^{29}$ Oxygen-free survival was higher in the hydrocortisone-treated group, especially in infants without antenatal steroids. Hypotension after recruitment was reduced by hydrocortisone. There was no difference in gastrointestinal perforation between the groups, however as many as $82 \%$ of all recruited infants received ibuprofen. This study showed more positive effects of hydrocortisone prophylaxis than the large trial by Watterberg et al and the trial by Peltoniemi et al. The authors explained the difference in outcome on the basis of the lower antenatal steroid used, the earlier start of hydrocortisone treatment and not allowing open-label use of steroids during the treatment period.

Five of the six studies reviewed above aimed to prevent CLD by early administration of hydrocortisone, whereas in the only retrospective study, hydrocortisone was prescribed to treat CLD. Dose and duration of hydrocortisone treatment varied considerably between the prevention and treatment studies, and four of the six studies were contaminated by open-label steroids prescription (dexamethasone) during and after hydrocortisone treatment (table 1 ).

\section{LONG-TERM FOLLOW-UP}

There have been two follow-up reports based on the initial 1972 hydrocortisone trial to alter the course or outcome in infants with respiratory distress syndrome. In 14 of the 16 children who died within 2 months of birth (seven in the hydrocortisone and seven in the placebo group) an autopsy revealed no differences in lung, liver, adrenal, thymus, heart and spleen pathology, attributable to steroid treatment. However, a significant association $\left(p<0.05 ; \chi^{2}\right.$ with Yates correction) was found between the occurrence of intraventricular haemorrhage and hydrocortisone treatment. ${ }^{30}$ At the age of 1 year, 24 survivors (12 in both groups) were examined. There was a tendency to slightly increased incidence of gross neurological and electroencephalographic abnormalities among the infants who received hydrocortisone, but the Griffiths Developmental Scale showed a normal developmental quotient in both groups. However, analysis of the subtests revealed a substantial difference in the results for the gross motor development with a lower mean score for the hydrocortisone group. ${ }^{31}$
Van de Heide et al retrospectively studied a group of 25 preterm infants treated with high-dose hydrocortisone. At the age of 5-7 years this group did not differ from a non-treated control group (matched for gestational age, birth weight, severity of infant respiratory distress syndrome, intraventricular haemorrhage and period of admission) with regard to neurological outcome, psychomotor development or school performance. Infants treated with dexamethasone required more special school education and had worse neurological outcome compared with controls. ${ }^{26}$

Of 294 survivors, 252 (86\%) infants who participated in Watterberg and colleagues' prophylactic hydrocortisone trial were evaluated at the age of 20.0 (SD 2.1) months by certified examiners masked to treatment assignment. The incidence of cerebral palsy was similar as was the mean Mental and Physical Developmental Index, estimated with the Bayley Scales of Infant Development II. However, a significantly smaller percentage of infants treated with hydrocortisone had a Mental Developmental Index below 70 (27\% in the hydrocortisone group vs $37 \%$ in the placebo group, $p=0.017$ ). ${ }^{32}$

In a follow-up study from our own institute, 23 preterm-born children (mean (SD) gestational age 28 (2) weeks), who received hydrocortisone treatment for chronic lung disease (starting dose of $5 \mathrm{mg} / \mathrm{kg} /$ day, tapered over a minimum of 3 weeks) in the neonatal period and 35 children (mean gestational age 30.4 (1.5) weeks) not treated with hydrocortisone during the neonatal period, were evaluated with quantitative MRI and neurocognitive assessment at the age of 8 years. Hydrocortisone treatment was introduced at a median age of 18 days (range 4-43 days) and none of the children was treated with dexamethasone at a later stage, thus avoiding contamination of the outcome results. We found that the children treated with hydrocortisone and the non-treated children had similar volumes of grey matter, white matter, cerebral fluid and hippocampus. The Wechsler Intelligence Scale for Children-Revised scores were within the normal range for both groups, with no difference between the groups. ${ }^{33}$ A subgroup of these patients underwent proton magnetic resonance spectroscopy of the hippocampus ${ }^{34}: 18$ hydrocortisone-treated infants (mean gestational age 27.9 (1.7) weeks) were compared with 19 non-treated preterm infants (mean gestational age 30.6 (1.7) weeks). Although the infants treated with hydrocortisone were younger, lighter and sicker than their counterparts, who were not treated with steroids, there was no difference in the $N$-acetyl aspartate/(choline and (phospho) creatine) ratios between the two groups at a median age of 8.4 years.

An extended group of children born preterm, including the above mentioned population, was evaluated recently. ${ }^{35}$ In all, 62 preterm-born children treated with hydrocortisone for CLD during the neonatal period were compared with 164 children who did not receive steroids during their admission to the neonatal intensive care unit. The hydrocortisone-treated children had a lower gestational age as well as lower birth weight, and were sicker than the non-steroid-treated children. To better deal with these group differences, adjustments were made for gestational age, birth weight, gender, need for mechanical ventilation and small for gestational age. The adjusted mean IO, and visual-motor integration and memory test results, were similar in the hydrocortisone-treated and non-treated groups. The incidence of cerebral palsy was also similar, and there was no difference in motor function between the two groups, as assessed with the Movement Assessment Battery for Children. All children had a brain MRI, and the occurrence of brain lesions 
as well as mean midsagittal corpus callosum areas were similar in the two groups of children.

Karemaker et al reported a retrospective matched-cohort study on long-term effects on behaviour and motor skills in school age (7-10 years) children who received either dexamethasone or hydrocortisone for CLD. A non-treated control group and a group treated only antenatally with betamethasone were included in the analysis. All groups were matched for gestational age, birth weight, gender, grade of respiratory distress syndrome and rate of peri/intraventricular haemorrhage. The children treated with dexamethasone had more neuromotor problems than the children in the control and betamethasone groups, whereas the outcomes in children treated with hydrocortisone did not differ from these two groups. $^{36}$

\section{DISCUSSION}

Compared with preterm infants not treated with hydrocortisone, no differences have been found in neurocognitive or motor outcome, or in the incidence of brain abnormalities on MRI, after hydrocortisone treatment for bronchopulmonary dysplasia in long-term follow-up studies at 5-8 years of age. ${ }^{263-36}$ Importantly these children were treated exclusively with hydrocortisone and there was no contamination by later prescription of dexamethasone. However, the patient population was retrospectively enrolled and hydrocortisone administration did not commence until after the first postnatal week. In the single follow-up study of prophylactic hydrocortisone for prevention of CLD, a large percentage of children were treated with open-label dexamethasone during or after the study period..$^{32}$ Open-label contamination can result in the treatment and placebo groups becoming more similar than intended, making it difficult to detect a difference in outcome among the two groups. In addition, open-label contamination will also increase the steroid dose received by babies in the index treatment arm, making interpretation even more difficult.

There may be several explanations why hydrocortisone treatment might not be associated with long-term neurological deficits, whereas dexamethasone is. Dexamethasone is a synthetic glucocorticoid with a 25-30 times higher antiinflammatory action than hydrocortisone. The typical dose of hydrocortisone is much lower than that of dexamethasone in most studies. This is also reflected in the fewer short-term neonatal complications with the use of hydrocortisone as compared with dexamethasone. ${ }^{26}$

In the brain, dexamethasone binds preferentially to the glucocorticoid receptor, ${ }^{37}$ and hydrocortisone binds preferentially to the mineralocorticoid receptor. Animal studies have shown that activation of the glucocorticoid receptor leads to adverse neuronal effects. ${ }^{38}{ }^{39}$ In a neuronal cell culture model, stimulation of the glucocorticoid receptor, such as occurs with (high-dose) dexamethasone treatment, promotes apoptosis of the granular cells in the hippocampus, whereas stimulation of the mineralocorticoid receptor, such as with (low-dose) hydrocortisone treatment, is protective against apoptosis. The opposing actions of mineralocorticoid receptor and glucocorticoid receptor on neuronal survival result from their ability to differentially influence the expression of members of the bcl-2 gene family (major regulatory components of the apoptotic pathway). ${ }^{40}$ Yet another mechanism may have a role. The enzyme 11ß-hydroxysteroid dehydrogenase type 2, which catalyses rapid inactivation of cortisol to inert 11-keto derivates, is abundant in the developing brain. It may protect the developing nervous system against the deleterious consequences of glucocorticoid overexposure such as in early hydrocortisone treatment. ${ }^{41}{ }^{42}$ This hypothesis needs further exploration.

The biologic half-life of dexamethasone is 36-72 $\mathrm{h}$ in contrast with that of 8-12 $\mathrm{h}$ for hydrocortisone. ${ }^{43}$ There may be less risk of accumulation of medication with hydrocortisone. The preservative agent in dexamethasone, which is used to control microbial and oxidative degeneration, is sodium bisulphite. Exposure of a neuronal cell line (rat mesencephalic cells) to high levels of sulphite induced a time-dependent decrease in viability. ${ }^{44}$ Sulphites have been shown to be toxic in vitro to cultures of neurons and in vivo to the brains of 3-5-day-old mouse pups. ${ }^{45}$

A recent study showed that a lower dose of dexamethasone than generally used $(0.89 \mathrm{mg} / \mathrm{kg} / 10$ days $)$ is also effective in facilitating extubation and shortening of duration of intubation among ventilator-dependent extremely low birthweight infants at a median treatment age of 23 days. ${ }^{46}$ There was little evidence for reduction in either the mortality rate or the rate of oxygen dependency at 36 weeks. Follow-up showed no difference in outcome in the treated and non-treated groups at 2 years of age, but doses of dexamethasone varied widely within and between the two groups. In addition, there was a high rate of contamination with open-label dexamethasone treatment in the placebo group. ${ }^{47}$

One could argue that the infants in the hydrocortisone follow-up studies were at a more advanced gestational age when receiving the medication than in most dexamethasone studies. The first report on the negative long-term effects of postnatal dexamethasone use by Yeh et $a l^{9}$ was on children with a mean gestational age of 29.8 (2.3) weeks, and Shinwell and coworkers also studied infants with a mean gestational age of 29.2 (2.6) weeks when receiving dexamethasone. ${ }^{10}$ A possible factor is that in the late treatment hydrocortisone follow-up studies, the infants were already 2 weeks postnatal, whereas in these dexamethasone studies the infants received the medication shortly after birth. Still in one study, there were considerably more cases of cerebral palsy and abnormal neurological examinations at 1 year of age after a 42-day course of dexamethasone started after 2 weeks of age. ${ }^{11}$ Another study included 22 infants (birth weight $<1250 \mathrm{~g}$ and gestational age $<30$ weeks) who survived to 15 years and were ventilatordependent at 2 weeks of age. Nine received a 42-day course of dexamethasone, eight received an 18 day-course of dexamethasone and five infants did not receive steroids. The authors concluded that the 42-day course was associated with improved long-term neurodevelopmental outcome, however, the number of patients in each subgroup was very small. ${ }^{48}$

In two early treatment (prophylactic) hydrocortisone studies, enrolment stopped because of an increased incidence of spontaneous gastrointestinal perforation with combined hydrocortisone-indometacin treatment. ${ }^{27} 28$ A cohort study of adult patients who were treated with corticosteroids combined with non-steroidal anti-inflammatory drugs reported a more than twofold increased risk of gastrointestinal bleeding when compared with the use of corticosteroid alone. ${ }^{49}$ In very low birthweight infants, low-dose hydrocortisone alone (in the absence of co-treatment with prophylactic indometacin) did not lead to gastrointestinal perforation. ${ }^{50}$ Peltoniemi et al found that pretreatment serum cortisol levels were associated with treatment effects and with the risk of intestinal perforations. ${ }^{28}$ Hallman et al advocated the need to modify the dosage, limit the drug-drug interactions and perhaps above all scrutinise patient selection in corticosteroid trials on very high-risk infants in early life by incorporating pretreatment cortisol 
measurements in the decision making-although this suggestion was based on only four patients in the Peltoniemi study. ${ }^{51}$ Treatment with hydrocortisone beyond 2 weeks of postnatal age will avoid the interactive effect of hydrocortisone with indometacin, therefore reducing the risk of gastrointestinal perforation considerably. Although two publications reported an increased risk of disseminated Candida infections in infants treated with hydrocortisone, ${ }^{52}$ this was not confirmed by other studies. ${ }^{18} 27$

\section{CONCLUSIONS}

We should aim for the lowest dose and shortest course of the least toxic steroid that facilitates weaning off the ventilator and protects against CLD. Currently, the clinical choice of steroids, dexamethasone or hydrocortisone is largely based on local culture and training, rather than on sufficient evidence from direct comparisons. In terms of quantitative published evidence there is equipoise. Claims for either of the steroids that randomised comparison would expose children to excess (avoidable) risks or would withhold the presumably best treatment to some children are not founded on direct evidence. However, neonatology as a discipline should decide whether it is necessary to make this randomised direct comparison between dexamethasone and hydrocortisone, or whether the available publications on long-term neurodevelopmental outcome provide sufficient evidence to justify a shift from dexamethasone towards hydrocortisone prescription for the treatment of CLD in the near future.

\section{Competing interests: None.}

\section{REFERENCES}

1. Committee on Obstetric Practice. ACOG committee opinion: antenatal corticosteroid therapy for fetal maturation. Obstet Gynecol 2002;99:871-3.

2. Barrington KJ. Hazards of systemic steroids for ventilator-dependent preterm infants: what would a parent want? CMAJ 2001;165:33-4.

3. Thebaud B, Lacaze-Masmonteil T, Watterberg K. Postnatal glucocorticoids in very preterm infants: "the good, the bad, and the ugly"? Pediatrics 2001;107:413-5.

4. Finer NN, Craft A, Vaucher YE, et al. Postnatal steroids: short-term gain, long-term pain? J Pediatr 2000;137:9-13.

5. Jobe AH. Postnatal corticosteroids for preterm infants-do what we say, not what we do. N Engl J Med 2004;350:1349-51.

6. Shinwell ES. Current dilemmas in postnatal steroid therapy for chronic lung disease in preterm infants. Biol Neonate 2003;84:96-100.

7. Mammel MC, Green TP, Johnson DE, et al. Controlled trial of dexamethasone therapy in infants with bronchopulmonary dysplasia. Lancet 1983:1:1356-8.

8. Hack M, Fanaroff AA. Outcomes of children of extremely low birthweight and gestational age in the 1990s. Early Hum Dev 1999;53:193-218.

9. Yeh TF, Lin YJ, Huang CC, et al. Early dexamethasone therapy in preterm infants: a follow-up study. Pediatrics 1998;101:E7.

10. Shinwell ES, Karplus M, Reich D, et al. Early postnatal dexamethasone treatment and increased incidence of cerebral palsy. Arch Dis Child Fetal Neonatal Ed 2000;83:F177-181.

11. O'Shea TM, Kothadia JM, Klinepeter KL, et al. Randomized placebo-controlled trial of a 42-day tapering course of dexamethasone to reduce the duration of ventilator dependency in very low birth weight infants: outcome of study participants at 1-year adjusted age. Pediatrics 1999:104:15-21.

12. Shinwell ES, Karplus M, Bader D, et al. Neonatologists are using much less dexamethasone. Arch Dis Child Fetal Neonatal Ed 2003:88:F432-3.

13. American Academy of Pediatrics, Committee on Fetus and Newborn. Postnatal corticosteroids to treat or prevent chronic lung disease in preterm infants. Pediatrics 2002;109:330-8.

14. Finer NN, Powers RJ, Ou CH, et al. Prospective evaluation of postnatal steroid administration: a 1-year experience from the California Perinatal Quality Care Collaborative. Pediatrics 2006;117:704-13.

15. Short EJ, Klein NK, Lewis BA, et al. Cognitive and academic consequences of bronchopulmonary dysplasia and very low birth weight: 8-year-old outcomes. Pediatrics 2003;112:e359.

16. Yeh TF, Lin YJ, Lin HC, et al. Outcomes at school age after postnatal dexamethasone therapy for lung disease of prematurity. N Engl J Med 2004:350:1304-13.

17. Parikh NA, Lasky RE, Kennedy KA, et al. Postnatal dexamethasone therapy and cerebral tissue volumes in extremely low birth weight infants. Pediatrics 2007:119:265-72
18. Ng PC, Lee $\mathrm{CH}$, Bnur FL, et al. A double-blind, randomized, controlled study of a "stress dose" of hydrocortisone for rescue treatment of refractory hypotension in preterm infants. Pediatrics 2006;117:367-75

19. Seri I. Hydrocortisone and vasopressor-resistant shock in preterm neonates. Pediatrics 2006;117:516-8.

20. Aucott SW. Hypotension in the newborn: who needs hydrocortisone? J Perinatol 2005:25:77-8.

21. Lattimore KA, Vazquez DM, Barks JD, et al. 12 month developmental outcome in infants treated with hydrocortisone for refractory hypotension. Presented at the Society for Pediatric Research meeting, Toronto, Canada, 2007. E-PAS: 617934.9.

22. Baden $\mathbf{M}$, Bauer CR, Colle $\mathrm{E}$, et al. A controlled trial of hydrocortisone therapy in infants with respiratory distress syndrome. Pediatrics 1972:50:526-34.

23. Watterberg KL, Gerdes JS, Gifford KL, et al. Prophylaxis against early adrenal insufficiency to prevent chronic lung disease in premature infants. Pediatrics 1999:104:1258-63.

24. Watterberg KL, Scott SM. Evidence of early adrenal insufficiency in babies who develop bronchopulmonary dysplasia. Pediatrics 1995:95:120-5.

25. Watterberg KL, Demers LM, Scott SM, et al. Chorioamnionitis and early lung inflammation in infants in whom bronchopulmonary dysplasia develops. Pediatrics 1996:97:210-5

26. Heide-Jalving $\mathbf{M}$, Kamphuis PJ, van der Laan MJ, et al. Short- and long-term effects of neonatal glucocorticoid therapy: is hydrocortisone an alternative to dexamethasone? Acta Paediatr 2003:92:827-35.

27. Watterberg KL, Gerdes JS, Cole CH, et al. Prophylaxis of early adrenal insufficiency to prevent bronchopulmonary dysplasia: a multicenter trial. Pediatrics 2004:114:1649-57.

28. Peltoniemi 0, Kari MA, Heinonen K, et al. Pretreatment cortisol values may predic responses to hydrocortisone administration for the prevention of bronchopulmonary dysplasia in high-risk infants. J Pediatr 2005;146:632-7.

29. Bonsante $\mathbf{F}$, Latorre G, lacobelli $\mathbf{S}$, et al. Early low-dose hydrocortisone in very preterm infants: a randomized, placebo-controlled trial. Neonatology 2007;91:21721.

30. Taeusch HW Jr, Wang NS, Baden N, et al. A controlled trial of hydrocortisone therapy in infants with respiratory distress syndrome: II. Pathology. Pediatrics 1973;52:850-4.

31. Fitzhardinge $\mathbf{P M}$, Eisen $\mathrm{A}$, Lejtenyi $\mathrm{C}$, et al. Sequelae of early steroid administration to the newborn infant. Pediatrics 1974:53:877-83.

32. Watterberg KL, Shaffer ML, Mishefske MJ, et al. Growth and neurodevelopmental outcomes after early low-dose hydrocortisone treatment in extremely low birth weight infants. Pediatrics 2007:120:40-8.

33. Lodygensky GA, Rademaker K, Zimine S, et al. Structural and functional brain development after hydrocortisone treatment for neonatal chronic lung disease. Pediatrics 2005;116:1-7

34. Rademaker KJ, Rijpert M, Uiterwaal CS, et al. Neonatal hydrocortisone treatment related to $1 \mathrm{H}-\mathrm{MRS}$ of the hippocampus and short-term memory at school age in preterm born children. Pediatr Res 2006;59:309-13.

35. Rademaker KJ, Uiterwaal CS, Groenendaal F, et al. Neonatal hydrocortisone treatment: neurodevelopmental outcome and MRI at school age in preterm-born children. J Pediatr 2007;150:351-7.

36. Karemaker $\mathbf{R}$, Heijnen CJ, Veen $\mathrm{S}$, et al. Differences in behavioral outcome and motor development at school age after neonatal treatment for chronic lung disease with dexamethasone versus hydrocortisone. Pediatr Res 2006;60 745-50.

37. De Kloet ER, Vreugdenhil E, Oitzl MS, et al. Brain corticosteroid receptor balance in health and disease. Endocr Rev 1998:19:269-301.

38. Sapolsky RM, Uno H, Rebert CS, et al. Hippocampal damage associated with prolonged glucocorticoid exposure in primates. J Neurosci 1990;10:2897-902.

39. Uno H, Tarara R, Else JG, et al. Hippocampal damage associated with prolonged and fatal stress in primates. J Neurosci 1989;9:1705-11.

40. Almeida OF, Conde GL, Crochemore C, et al. Subtle shifts in the ratio between proand antiapoptotic molecules after activation of corticosteroid receptors decide neuronal fate. FASEB J 2000;14:779-90.

41. Holmes MC, Seckl JR. The role of 11 beta-hydroxysteroid dehydrogenases in the brain. Mol Cell Endocrinol 2006:248:9-14.

42. Holmes MC, Sangra M, French KL, et al. 11 beta-Hydroxysteroid dehydrogenase type 2 protects the neonatal cerebellum from deleterious effects of glucocorticoids. Neuroscience 2006;137:865-73.

43. Taketomo CK, Hodding JH, Kraus DM. Pediatric dosage handbook, 11th edn Hudson, OH: Lexi-Comp, 2004.

44. Reist M, Marshall KA, Jenner P, et al. Toxic effects of sulphite in combination with peroxynitrite on neuronal cells. J Neurochem 1998;71:2431-8.

45. Baud 0, Laudenbach V, Evrard P, et al. Neurotoxic effects of fluorinated glucocorticoid preparations on the developing mouse brain: role of preservatives. Pediatr Res 2001;50:706-11.

46. Doyle LW, Davis PG, Morley CJ, et al. Low-dose dexamethasone facilitates extubation among chronically ventilator-dependent infants: a multicenter, international, randomized, controlled trial. Pediatrics 2006:117:75-83

47. Doyle LW, Davis PG, Morley CJ, et al. Outcome at 2 years of age of infants from the DART study: a multicenter, international, randomized, controlled trial of low-dose dexamethasone. Pediatrics 2007:119:716-21.

48. Gross SJ, Anbar RD, Mettelman B. Follow-up at 15 years of preterm infants from a controlled trial of moderately early dexamethasone for the prevention of chronic lung disease. Pediatrics 2005:115:681-7. 
49. Nielsen GL, Sorensen HT, Mellemkjoer $L$, et al. Risk of hospitalization resulting from upper gastrointestinal bleeding among patients taking corticosteroids: a registerbased cohort study. Am J Med 2001;111:541-5.

50. Caeymaex L, Gatel P, Castel C, et al. Low-dose hydrocortisone alone does not lead to gastro-intestinal perforation in ELBW infants. Presented at the Society for Pediatric Research meeting, San Francisco, CA, USA, E-PAS 2006;50: 2861.220.
51. Hallman M, Peltoniemi O, Saarela T. Early neonatal hydrocortisone: study rather than treat. Pediatrics 2006;118:2540-2.

52. Ramanathan R, Siassi B, Sardesai S, et al. Dexamethasone versus hydrocortisone for hypotension refractory to high dose inotropic agents and incidence of candida infection in extremely low birth weight infants. Pediatr Res 2006;39:A240.

53. Botas CM, Kurlat I, Young SM, et al. Disseminated candidal infections and intravenous hydrocortisone in preterm infants. Pediatrics 1995:95:883-7.

\section{FUNDING AVAILABLE FOR RESEARCH PROJECTS}

The Committee on Publication Ethics (COPE) has established a Grant Scheme to fund research in the field of publication ethics. The Scheme is designed to provide financial support to any member of COPE for a defined research project that is in the broad area of the organisation's interests, and specifically in the area of ethical standards and practice in biomedical publishing. The project should have a specific goal and be intended to form the kernel of a future publication.

A maximum sum of $f 5000$ will be allocated to any one project, but applications for smaller sums are welcomed.

The terms and conditions of the Grant are as follows:

- At least one of the applicants must be a member of COPE.

- Calls for applications will be made twice a year with closing dates of 1 December and 1 June. An electronic version of the application form must be sent to the Administrator no later than $12 \mathrm{pm}$ (noon GMT) on the closing date for consideration by COPE Council.

- The application must contain a lay summary of the project, a definition of the question to be posed, sufficient methodological detail to allow assessment of the viability of the project, a clear timeline and a definition of the likely deliverables. A full justification for the sum requested must accompany the application.

- A report on the progress of the research should be presented within one year of the award and at the end of the project. The grant must be used within two years from the date of award, and balance sheets must be forwarded annually. These should be sent to the Administrator. Any remaining funds after two years must be returned.

- It is anticipated that the work stemming from the project will be presented at one of COPE's annual seminar meetings within 2-3 years of the award. Such data may also be published in peer-reviewed journals. Any publications or related presentations at meetings by the recipient emanating in part or whole from COPE's support should be duly acknowledged and copies sent to the Administrator.

Applications are reviewed by a COPE sub-committee. Applicants will be advised of a decision as soon as practicable after the deadline date.

An application form can be obtained by contacting Linda Gough, COPE administrator, at LGough@ bmj.com or 0207383 6602. For more information on COPE, see http://www.publicationethics.org.uk/

The closing date for receipt of applications is 1 December 2007 or 1 June 2008. 